The Annals of Probability

2002, Vol. 30, No. 2, 483-508

\title{
FINITE-DIMENSIONAL APPROXIMATION OF THE SELF-DIFFUSION COEFFICIENT FOR THE EXCLUSION PROCESS
}

\author{
By C. LANDim, ${ }^{1}$ S. Olla And S. R. S. VARADHAN ${ }^{2}$ \\ IMPA and CNRS UPRES, Université de Cergy CNRS UPRES and \\ Courant Institute, New York University
}

\begin{abstract}
We show that for the symmetric simple exclusion process on $\mathbb{Z}^{d}$ the self-diffusion coefficient of a tagged particle is stable when approximated by simple exclusion processes on large periodic lattices. The proof depends on a similar stability property of the asymptotic variance of additive functionals of mean 0 . This requires establishing a property for the Dirichlet space known as the Liouville-D property.
\end{abstract}

1. Introduction. In the early 1980s, Kipnis and Varadhan proved an invariance principle for the position of a tagged particle in a symmetric simple exclusion process in equilibrium (cf. [6]). Their proof relied on a general central limit theorem for additive functionals of a reversible Markov process that they established. Time reversibility and translation invariance of the system are the basic ingredients of this method, and, in principle, it can be applied to any system with these two symmetries. Later it was extended to nonreversible processes that satisfy a strong sector condition (cf. [10] and [13]).

The (effective) diffusion matrix of the limiting Brownian motion is a function $D(\alpha)$ of the density $\alpha$ of the particles, and in the reversible case it is given by a variational formula.

This method works directly in infinite systems, and it raises the natural question of the stability of the effective diffusion matrix under finite-dimensional approximations. More precisely, consider a finite-dimensional version of the same process on the torus $\{-N, \ldots, 0, \ldots, N\}^{d}$ (i.e., with periodic boundary conditions, preserving in this manner the translation symmetry). Since we want to work with an ergodic process, we also fix the total number $K$ of particles. Consider now a tagged particle in this finite system. If $N$ is much larger than the size of a single jump, the motion of the tagged particle has a unique canonical lifting to $\mathbb{Z}^{d}$. We get in this manner a process $X_{N}(t)$ with values in $\mathbb{Z}^{d}$. Let us denote by $D_{[N, K]}$

Received October 2000; revised June 2001.

${ }^{1}$ Supported in part by CNPq Grant 300358/93-8, FAPERJ Grant E26/150.940/99 and PRONEX Grant 41.96.0923.00.

${ }^{2}$ Supported by NSF Grant DMS-98-03140.

AMS 2000 subject classification. $60 \mathrm{~K} 35$.

Key words and phrases. Self-diffusion, tagged particle, exclusion process, Liouville property. 
the variance of the Brownian motion which is the limit of the scaled process $\varepsilon X_{N}\left(\varepsilon^{-2} t\right)$ as $\varepsilon \rightarrow 0$. We expect that

$$
\lim _{\substack{N \rightarrow \infty \\ K /(2 N)^{d} \rightarrow \alpha}} D_{[N, K]}=D(\alpha) .
$$

Related questions arise in other contexts, for example, the effective conductivity in percolation regime (cf. [1] and [4]) and fluctuations in massless interface models (cf. [2]).

In general, it is easy to prove that

$$
\limsup _{\substack{N \rightarrow \infty \\ K /(2 N)^{d} \rightarrow \alpha}} D_{[N, K]} \leq D(\alpha) .
$$

In this paper we prove equality (1.1) for symmetric simple exclusion processes on $\mathbb{Z}^{d}$.

A related problem that is somewhat easier to handle is the scaling behavior of an additive functional $A(t)=\int_{0}^{t} V(\eta(s)) d s$, where $V$ is a suitable local function that depends on the configuration at a few sites. There is again a limiting Brownian motion for $\varepsilon A\left(\varepsilon^{-2} t\right)$ with variance $\sigma_{N, K}^{2}$. The problem is to show that

$$
\lim _{\substack{N \rightarrow \infty \\ K /(2 N)^{d} \rightarrow \alpha}} \sigma_{N, K}^{2}=\sigma^{2}(\alpha),
$$

where $\sigma^{2}(\alpha)$ is the corresponding variance in the infinite-volume situation where the central limit theorem can be established directly. In this context the nontrivial part is the inequality

$$
\limsup _{\substack{N \rightarrow \infty \\ K /(2 N)^{d} \rightarrow \alpha}} \sigma_{N, K}^{2} \leq \sigma^{2}(\alpha) .
$$

The proofs depend on approximating the solutions of the infinite-dimensional variational problem for the limiting variance by solutions of the corresponding finite-dimensional problem.

A basic step in this approximation is to prove a certain regularity property of the Dirichlet form associated with the simple exclusion process. Let us describe this regularity property in a more general context.

Suppose we have a collection of invertible measure-preserving transformations $\left\{T_{z}\right\}$ on some probability space $(\Omega, \mathcal{F}, \mu)$. Then we can define a Dirichlet form

$$
D(u)=\frac{1}{2} \int_{\Omega} \sum_{z}\left[u\left(T_{z} \omega\right)-u(\omega)\right]^{2} d \mu
$$

corresponding to the generator

$$
L u=\sum_{z}\left[u\left(T_{z} \omega\right)-u(\omega)\right]
$$


of a process consisting of random stirrings. If we define $v_{z}(\omega)=\left(\nabla_{z} u\right)(\omega)=$ $u\left(T_{z} \omega\right)-u(\omega)$, there is natural map

$$
u \rightarrow \delta u=\left\{v_{z}(\omega)\right\} \in \bigoplus_{z} L_{2}(\mu) .
$$

The problem is to characterize the closure of the range of this map by relations that remain stable under suitable approximations done with finitely supported functions.

In Section 3 we prove this regularity property for the symmetric simple exclusion processes, and in Section 4 for the version with a tagged particle. The main tool in the proof is duality, which allows us to reduce the problem to a finitedimensional situation (cf. [8] and [11]).

A similar question arises in the context of a hydrodynamic limit for nongradient models (cf. [5], Appendix 3, and [14]). But there the approximation is needed in a different norm and the proof relies usually on a suitable estimate of the spectral gap for the corresponding dynamics in a finite volume.

2. Notation and results. Let us fix a symmetric, finite-range probability measure $p(\cdot)$ on $\mathbb{Z}^{d}: p(0)=0, p(z)=p(-z)$ and $p(z)=0$ for $|z|$ large. Let us assume that the random walk with transition probability $p(\cdot)$ is irreducible; that is, the finite set $\{z: p(z)>0\}$ generates the group $\mathbb{Z}^{d}$. Consider the symmetric simple exclusion process associated with $p(\cdot)$. This is the Markov process on $\mathcal{X}=\{0,1\}^{\mathbb{Z}^{d}}$ whose generator $L$ acting on cylinder functions $f$ is given by

$$
\begin{aligned}
(L f)(\eta) & =\sum_{x, y \in \mathbb{Z}^{d}} p(y-x) \eta(x)[1-\eta(y)]\left[f\left(\sigma^{x, y} \eta\right)-f(\eta)\right] \\
& =\frac{1}{2} \sum_{x, y \in \mathbb{Z}^{d}} p(y-x)\left[f\left(\sigma^{x, y} \eta\right)-f(\eta)\right] .
\end{aligned}
$$

Here and below, a configuration of particles in $\mathbb{Z}^{d}$, that is, a point in $\mathcal{X}$, will be denoted by $\eta$. In particular, for $x$ in $\mathbb{Z}^{d}, \eta(x)$ is equal to 1 if the site $x$ is occupied and 0 otherwise. Moreover, for a configuration $\eta$ and $x, y$ in $\mathbb{Z}^{d}, \sigma^{x, y} \eta$ is the configuration obtained from $\eta$ by exchanging the occupation variables $\eta(x)$ and $\eta(y)$ :

$$
\left(\sigma^{x, y} \eta\right)(z)= \begin{cases}\eta(y), & \text { if } z=x, \\ \eta(x), & \text { if } z=y, \\ \eta(z), & \text { otherwise. }\end{cases}
$$

Note that $\sigma^{x, y}=\sigma^{y, x}$ and we can think of either one as being $\sigma^{e}$, where $e$ is an edge or a bond connecting $x$ and $y$. Fix $0 \leq \alpha \leq 1$ and denote by $v_{\alpha}$ the Bernoulli product measure on $\mathcal{X}$. This is the probability measure on $\mathcal{X}$ obtained by placing a particle with probability $\alpha$ at each site $x$, and independently for different sites. It is easy to check that the one-parameter family of probability measures 
$\left\{v_{\alpha}, 0 \leq \alpha \leq 1\right\}$ are reversible invariant measures for the process with generator $L$ on $\mathcal{X}$. The Dirichlet form is given by

$$
D(f)=\frac{1}{4} \int_{X} \sum_{x, y \in \mathbb{Z}^{d}} p(y-x)\left[f\left(\sigma^{x, y} \eta\right)-f(\eta)\right]^{2} d v_{\alpha} .
$$

We will think of $\mathbb{Z}^{d}$ as a connected graph with an edge $e$ connecting $x, y$ being present whenever $p(y-x)>0$. Since $p(\cdot)$ is symmetric, one can view $p(y-x)$ as defined on edges with $p(e)=p(y-x)$. Denote by $\mathbb{E}$ the set of such edges. For each local function $f$, denote by $\delta f$ the gradient obtained from $f$. This is the collection $\left\{\left(\nabla_{e} f\right)(\eta)\right\}$ of functions on $\mathcal{X}$ defined for $e=(x, y) \in \mathbb{E}$ by

$$
\left(\nabla_{e} f\right)(\eta)=f\left(\sigma^{x, y} \eta\right)-f(\eta) .
$$

Since $\sigma^{x, y}=\sigma^{y, x}$ we can define $\sigma^{e}$ for $e \in \mathbb{E}$. If $e_{1}, \ldots, e_{n}$ are $n$ edges in $\mathbb{E}$ such that $\sigma^{e_{1}} \cdots \sigma^{e_{n}}$ is the identity permutation, then

$$
\begin{aligned}
0 & =f\left(\sigma^{e_{1}} \cdots \sigma^{e_{n}} \eta\right)-f(\eta) \\
& =\sum_{j=1}^{n}\left[f\left(\sigma^{e_{j}} \cdots \sigma^{e_{n}} \eta\right)-f\left(\sigma^{e_{j+1}} \cdots \sigma^{e_{n}} \eta\right)\right] \\
& =\sum_{j=1}^{n}\left(\nabla_{e_{j}} f\right)\left(\sigma^{e_{j+1}} \cdots \sigma^{e_{n}} \eta\right) \\
& =\sum_{j=1}^{n} \sigma^{e_{j+1}} \cdots \sigma^{e_{n}}\left(\nabla_{e_{j}} f\right)(\eta) .
\end{aligned}
$$

It is understood here that for $j=n$ the expression is just $\left(\nabla_{e_{n}} f\right)(\eta)$. The above conditions are called the compatibility conditions for a gradient and they permit us to recognize when an arbitrary collection $\Phi=\left\{\Phi_{e}, e \in \mathbb{E}\right\}$ of $L^{2}\left(v_{\alpha}\right)$ functions could be a gradient. We say that such a collection is a formal gradient if each $\Phi_{e}$ is square integrable and whenever $e_{1}, \ldots, e_{n}$ is a finite set of edges in $\mathbb{E}$ such that $\sigma^{e_{1}} \cdots \sigma^{e_{n}}$ is equal to the identity permutation, we have

$$
\sum_{j=1}^{n} \sigma^{e_{j+1}} \cdots \sigma^{e_{n}} \Phi_{e_{j}}=0 .
$$

As before, for $j=n$ this expression is $\Phi_{e_{n}}$. We associate a Hilbert space with norm defined by

$$
\|\Phi\|_{\alpha}^{2}=\sum_{e \in \mathbb{E}} p(e)\left\langle\Phi_{e}^{2}\right\rangle_{\nu_{\alpha}} .
$$

Any $\Phi$ for which the norm is finite will be called a square-integrable formal gradient. Although we have defined $\nabla_{e} f$ only for edges $e=(x, y)$ with $p(y-x)>0$, 
if the random walk with transition probability $p(\cdot)$ is irreducible then knowing $\nabla_{e}$ for edges in $\mathbb{E}$ determines it uniquely for all edges $(x, y)$. The Dirichlet forms corresponding to different irreducible finitely supported probabilities $p(\cdot)$ are equivalent. For qualitative statements we could take as a Dirichlet form simply

$$
\frac{1}{4} \int \sum_{\substack{x, y \\ y-x \in F}}\left|f\left(\sigma^{x, y} \eta\right)-f(\eta)\right|^{2} d v_{\alpha}
$$

where $F$ is any symmetric set that generates $\mathbb{Z}^{d}$ as a group. The natural question in this context is whether any square-integrable formal gradient can be approximated by actual gradients in the $L^{2}$ sense. That is, for each $\Phi=\left\{\Phi_{e}\right\}$, does there exist a sequence of local functions $f_{n}$ such that

$$
\lim _{n \rightarrow \infty}\left\|\Phi-\delta f_{n}\right\|_{\alpha}=0 ?
$$

The next result states that this is indeed possible.

THEOREM 2.1. For the symmetric exclusion process on $\mathbb{Z}^{d}$, any squareintegrable formal gradient can be approximated by gradients of local functions in the sense of (2.5).

COROLlARY 2.2. For a symmetric simple exclusion process on $\mathbb{Z}^{d}$, the limit (1.2) is valid for any local function $V$ which has mean 0 with respect to every $v_{\alpha}$.

To study the evolution of a tagged particle, we must consider simple exclusion on the lattice $\mathbb{Z}_{*}^{d}=\mathbb{Z}^{d}-\{0\}$. We have analogous results in that case. Of course, in dealing with simple exclusion on $\mathbb{Z}_{*}^{d}, \mathbb{E}$ contains only edges $(x, y)$ such that $y-x \in F$ and $x, y \in \mathbb{Z}_{*}^{d}$ and the Dirichlet form is now given by

$$
D(f)=\frac{1}{4} \int_{x} \sum_{\substack{x, y \in \mathbb{Z}_{*}^{d} \\ y-x \in F}} p(y-x)\left[f\left(\sigma^{x, y} \eta\right)-f(\eta)\right]^{2} d \nu_{\alpha} .
$$

THEOREM 2.3. For the symmetric exclusion process (other than the onedimensional nearest neighbor walk) on $\mathbb{Z}_{*}^{d}$, any square-integrable formal gradient can be approximated by gradients of local functions in the sense of (2.5).

COROLlARY 2.4. For a symmetric simple exclusion process (other than the one-dimensional nearest neighbor walk) on $\mathbb{Z}_{*}^{d}$, the limit (1.2) is valid for any local function $V$ which has mean 0 with respect to every $v_{\alpha}$.

We will apply Theorem 2.3 in order to study the evolution of a tagged particle in the symmetric simple exclusion process. Let $\eta$ be an initial configuration with a particle at the origin: $\eta(0)=1$. Tag this particle and denote by $\eta_{t}$ and $X_{t}$ the 
state of the process and the position of the tagged particle, respectively, at time $t$. We shall refer to $\eta_{t}$ as the environment. Let $\xi_{t}$ be the state of the environment as seen from the tagged particle: $\xi_{t}=\tau_{X_{t}} \eta_{t}$. Here, for $x$ in $\mathbb{Z}^{d}$ and a configuration $\eta, \tau_{x}$ stands for the translation of $\eta$ by $x$ units: $\left(\tau_{x} \eta\right)(y)=\eta(x+y)$. Notice that the origin is always occupied (by the tagged particle) for the environment as seen from the tagged particle. For this reason, we shall consider the process $\xi_{t}$ as taking values in $\{0,1\}^{\mathbb{Z}_{*}^{d}}$.

While $X_{t}$ is not a Markov process due to the presence of the environment, $\left(X_{t}, \eta_{t}\right)$ and $\xi_{t}$ are Markov processes. A simple computation shows that the generator $\mathcal{L}$ of the Markov process $\xi_{t}$ is given by $\mathcal{L}=\mathscr{L}_{0}+\mathscr{L}_{\tau}$, where

$$
\begin{aligned}
\left(\mathcal{L}_{0} f\right)(\xi) & =\sum_{x, y \in \mathbb{Z}_{*}^{d}} p(y-x) \xi(x)[1-\xi(y)]\left[f\left(\sigma^{x, y} \xi\right)-f(\xi)\right] \\
& =\frac{1}{2} \sum_{x, y \in \mathbb{Z}_{*}^{d}} p(y-x)\left[f\left(\sigma^{x, y} \xi\right)-f(\xi)\right] \\
\left(\mathcal{L}_{\tau} f\right)(\xi) & =\sum_{z \in \mathbb{Z}_{*}^{d}} p(z)[1-\xi(z)]\left[f\left(\tau_{z} \xi\right)-f(\xi)\right] .
\end{aligned}
$$

The first part of the generator takes into account the jumps of the environment, while the second one corresponds to jumps of the tagged particle. In the above formula, $\tau_{z} \xi$ stands for the configuration where the tagged particle, sitting at the origin, is first transferred to site $z$ and then the entire configuration is translated by $-z$; that is, for $x \neq 0$,

$$
\left(\tau_{z} \xi\right)(x)= \begin{cases}0, & \text { if } x=-z \\ \xi(x+z), & \text { for } x \neq-z\end{cases}
$$

For $0 \leq \alpha \leq 1$, denote by $\mu_{\alpha}$ the Bernoulli product measure on $\mathcal{X}_{*}=\{0,1\}^{\mathbb{Z}_{*}^{d}}$. A simple computation shows that $\mu_{\alpha}$ is reversible for the Markov process $\xi_{t}$.

Kipnis and Varadhan [6] proved a central limit theorem for the position of the tagged particle when the environment process is in equilibrium with marginal distribution $\mu_{\alpha}$. They showed that $\varepsilon X_{t \varepsilon^{-2}}$ converges, as $\varepsilon \downarrow 0$, to a Brownian motion with self-diffusion coefficient $D(\alpha)$ that can be described as follows. Fix $\lambda$ in $\mathbb{R}^{d}$. Then the quadratic form $\langle\lambda, D(\alpha) \lambda\rangle$ is given by the variational formula

$$
\begin{array}{r}
\langle\lambda, D(\alpha) \lambda\rangle=\inf _{f}\left\{\sum_{z \in \mathbb{Z}_{*}^{d}} p(z)\left\langle[1-\xi(z)]\left\{\lambda z-T_{z} f\right\}^{2}\right\rangle_{\alpha}\right. \\
\left.+\sum_{x, y \in \mathbb{Z}_{*}^{d}} p(y-x)\left\langle\left\{T_{x, y} f\right\}^{2}\right\rangle_{\alpha}\right\} .
\end{array}
$$

In this formula the infimum is taken over all cylinder functions $f,\langle\cdot\rangle_{\alpha}$ stands for the expectation with respect to the measure $\mu_{\alpha}, T_{z}, T_{x, y}, x, y, z$ in $\mathbb{Z}_{*}^{d}$, are the operators defined by 


$$
\begin{aligned}
\left(T_{z} f\right)(\xi) & =[1-\xi(z)]\left\{f\left(\tau_{z} \xi\right)-f(\xi)\right\}, \\
\left(T_{x, y} f\right)(\xi) & =\left\{f\left(\sigma^{x, y} \xi\right)-f(\xi)\right\}
\end{aligned}
$$

and the second summation is performed over all bonds of $\mathbb{Z}_{*}^{d}$.

The diffusion coefficient $D(\alpha)$ can also be written as

$$
\langle\lambda \cdot D(\alpha) \lambda\rangle=(1-\alpha) \sum_{z \in \mathbb{Z}_{*}^{d}}(z \cdot \lambda)^{2} p(z)-2\left\|\psi_{\lambda}\right\|_{-1, \alpha}^{2},
$$

where $\psi_{\lambda}$ is the cylinder function given by

$$
\psi_{\lambda}(\xi)=\sum_{z \in \mathbb{Z}_{*}^{d}} p(z)(z \cdot \lambda)[1-\xi(z)]
$$

and $\left\|\psi_{\lambda}\right\|_{-1, \alpha}^{2}$ is the $H_{-1}$ norm of $\psi_{\lambda}$, which is given by

$$
\|\psi\|_{-1, \alpha}^{2}=\sup _{f}\left\{2\left\langle f, \psi_{\lambda}\right\rangle_{\alpha}-\langle f,(-\mathcal{L}) f\rangle_{\alpha}\right\}
$$

In this formula the supremum is carried over all cylinder functions $f$ in $\mathcal{X}_{*}$ and $\langle\cdot, \cdot\rangle_{\alpha}$ stands for the inner product in $L^{2}\left(\mu_{\alpha}\right)$.

Fix a positive integer $N$ and denote by $\mathbb{T}_{N}^{d}=\{-N, \ldots, N\}^{d}$ the discrete $d$-dimensional torus with $N$ and $-N$ identified, consisting of $(2 N)^{d}$ points. For the same transition probability $p(\cdot)$, one can consider the symmetric simple exclusion process evolving on $\mathbb{T}_{N}^{d}$. The state space is now $\mathcal{X}_{N}=\{0,1\}^{\mathbb{T}_{N}^{d}}$ and the generator $L_{N}$ acting on any function $f$ is given by

$$
\left(L_{N} f\right)(\eta)=\sum_{x, y \in \mathbb{T}_{N}^{d}} p(y-x) \eta(x)[1-\eta(y)]\left[f\left(\sigma^{x, y} \eta\right)-f(\eta)\right]
$$

In a similar way, we can examine the evolution of a tagged particle on the torus $\mathbb{T}_{N}^{d}$. The environment as seen from the tagged particle is a Markov process with generator $\mathscr{L}_{N}=\mathscr{L}_{0, N}+\mathscr{L}_{\tau, N}$, where

$$
\begin{aligned}
& \left(\mathcal{L}_{0, N} f\right)(\xi)=\sum_{x, y \in \mathbb{T}_{N, *}^{d}} p(y-x) \xi(x)[1-\xi(y)]\left[f\left(\sigma^{x, y} \xi\right)-f(\xi)\right], \\
& \left(\mathcal{L}_{\tau, N} f\right)(\xi)=\sum_{z \in \mathbb{T}_{N, *}^{d}} p(z)[1-\xi(z)]\left[f\left(\tau_{z} \xi\right)-f(\xi)\right] .
\end{aligned}
$$

Let the total number $K$ of particles be fixed and, of course, $1 \leq K \leq(2 N)^{d}$. A simple computation shows that the measure $\mu_{N, K}$, which is uniform over the configurations of $\mathcal{X}_{N, *}=\{0,1\}^{\mathbb{T}_{N, *}^{d} \text { with } K-1 \text { particles, is reversible with respect }}$ to $\mathcal{L}_{N}$. Since we are in a finite state space, it is not hard to prove an invariance principle for the motion $X_{t}^{N}$ of the tagged particle lifted to $\mathbb{Z}^{d}$ in this context: 
$\varepsilon X_{t \varepsilon^{-2}}^{N}$ converges as $\varepsilon \downarrow 0$ to a Brownian motion with diffusion coefficient $D_{N, K}$ given by

$$
\left\langle\lambda, D_{N, K} \lambda\right\rangle=\left(1-\frac{K-1}{(2 N)^{d}-1}\right) \sum_{z \in \mathbb{Z}_{*}^{d}}(z \cdot \lambda)^{2} p(z)-2\left\|\psi_{\lambda}\right\|_{-1, N, K}^{2},
$$

where $\left\|\psi_{\lambda}\right\|_{-1, N, K}^{2}$ stands for the $H_{-1}$ norm of $\psi_{\lambda}$ in $L^{2}\left(\mu_{N, K}\right)$, given by the variational formula

$$
\left\|\psi_{\lambda}\right\|_{-1, N, K}^{2}=\sup _{f}\left\{2\left\langle\psi_{\lambda}, f\right\rangle_{N, K}-\left\langle f,\left(-\mathcal{L}_{N}\right) f\right\rangle_{N, K}\right\} .
$$

Here the supremum is carried over all functions in $L^{2}\left(\mu_{N, K}\right)$.

The main result of this article concerns the behavior of the finite-volume diffusion coefficient $D_{N, K}$ as $N \uparrow \infty$ :

THEOREM 2.5. Fix $\alpha$ in $(0,1)$ and a sequence $K_{N}$ such that $K_{N} /(2 N)^{d}$ converges to $\alpha$ as $N \uparrow \infty$. Then

$$
\lim _{N \rightarrow \infty} D_{N, K_{N}}=D(\alpha) .
$$

We conclude this section with a sketch of the proof of Theorem 2.5. In view of formulas (2.7) and (2.11), Theorem 2.5 follows from the convergence of the $H_{-1}$ norms of $\psi_{\lambda}$. It is enough to show that

$$
\lim _{\substack{N \uparrow \infty \\ K_{N} /(2 N)^{d} \rightarrow \alpha}}\left\langle\psi_{\lambda},\left(-\mathcal{L}_{N}\right)^{-1} \psi_{\lambda}\right\rangle_{N, K_{N}}=\left\langle\psi_{\lambda},(-\mathcal{L})^{-1} \psi_{\lambda}\right\rangle_{\alpha} .
$$

Here, to emphasize the dependence of the $H_{-1}$ norm on the generators, we have denoted the $H_{-1}$ norms $\|\cdot\|_{-1, N, K}^{2}$ and $\|\cdot\|_{-1, \alpha}^{2}$, respectively, by $\left\langle\cdot,\left(-\mathcal{L}_{N}\right)^{-1} \cdot\right\rangle_{N, K}$ and $\left\langle\cdot,(-\mathcal{L})^{-1} \cdot\right\rangle_{\alpha}$.

The generator $\mathcal{L}$ has two pieces. The first one, $\mathcal{L}_{0}$, corresponds to jumps of the environment and the second one, $\mathcal{L}_{\tau}$, corresponds to jumps of the tagged particle. From Corollary 2.4 we conclude that

$$
\lim _{\substack{N \uparrow \infty \\ K_{N} /(2 N)^{d} \rightarrow \alpha}}\left\langle\psi_{\lambda},\left(-\mathcal{L}_{0, N}\right)^{-1} \psi_{\lambda}\right\rangle_{N, K_{N}}=\left\langle\psi_{\lambda},\left(-\mathcal{L}_{0}\right)^{-1} \psi_{\lambda}\right\rangle_{\alpha} .
$$

In Section 4 we prove, in addition, that for each $\varepsilon>0$, we may express the local function $\psi_{\lambda}$ as the sum of $\mathcal{L} g_{\varepsilon}+u_{\varepsilon}$, where $g_{\varepsilon}, u_{\varepsilon}$ are local functions, $u_{\varepsilon}$ has mean zero with respect to all invariant measures and $\left\|u_{\varepsilon}\right\|_{0,-1}^{2}=\left\langle u_{\varepsilon},\left(-\mathcal{L}_{0}\right)^{-1} u_{\varepsilon}\right\rangle_{\alpha}$ $\leq \varepsilon^{2}$. Since $\|\cdot\|_{-1} \leq\|\cdot\|_{0,-1}$ and (2.13) is obvious if we replace $\psi_{\lambda}$ by a local function of the form $\mathcal{L} f$, (2.13) follows from the previous decomposition and (2.14). Details are given in Section 4. 
3. Formal gradients of the symmetric exclusion process. We prove in this section Theorems 2.1 and 2.3 and Corollaries 2.2 and 2.4. The proofs rely on the duality properties of the symmetric exclusion process that we now explain. Let $\mathbb{X}$ be a countable set. For us it will be either $\mathbb{Z}^{d}$ or $\mathbb{Z}^{d}-\{0\}$. We denote by $\mathcal{X}=\{0,1\}^{\mathbb{X}}$ the set of configurations $\eta$ on $\mathbb{X}$. For $0 \leq \alpha \leq 1, v_{\alpha}$ is the Bernoulli product measure on $\mathcal{X}$ with $P[\eta(x)=1]=\alpha$ for each $x \in \mathbb{X}$. Averages or norms with respect to $v_{\alpha}$ will be denoted by the subscript $\alpha$. Let $\mathbb{E}$ be a collection of edges $e=(x, y)$ connecting some pairs of points in $\mathbb{X}$. Assume that the resulting graph is connected and that every vertex in it has a finite degree. There will also be given transition rates $\{p(x, y): \mathbb{X} \times \mathbb{X} \rightarrow[0, \infty)\}$ that are symmetric and satisfy $\sum_{y \in \mathbb{X}} p(x, y) \leq 1$ for every $x \in \mathbb{X}$. Moreover, $\mathbb{E}=\{e=(x, y): p(x, y)>0\}$. In our context $p(x, y)=p(y-x)$ for $x, y \in \mathbb{Z}^{d}$ or $x, y \in \mathbb{Z}_{*}^{d}$ as the case may be. We have a simple exclusion process on $\mathcal{X}$ with generator

$$
(\mathcal{L} f)(\eta)=\sum_{x, y \in \mathbb{X}} p(x, y) \eta(x)(1-\eta(y))\left[f\left(\sigma^{x, y} \eta\right)-f(\eta)\right]
$$

and Dirichlet form

$$
D(f)=\frac{1}{4} \sum_{x, y \in \mathbb{X}} p(x, y)\left\|f\left(\sigma^{x, y} \eta\right)-f(\eta)\right\|_{\alpha}^{2} .
$$

For each $n \geq 0$, denote by $\varepsilon_{n}$ the subsets of $\mathbb{X}$ with $n$ points. For each $A$ in $\varepsilon_{n}$, let $\Psi_{A}$ be the local function

$$
\Psi_{A}=\prod_{x \in A} \frac{\eta(x)-\alpha}{\sqrt{\alpha(1-\alpha)}}
$$

By convention, $\Psi_{\phi}=1$. It is easy to check that $\left\{\Psi_{A}, A \subset \mathbb{X}\right\}$ is an orthonormal basis of $L^{2}\left(v_{\alpha}\right)$. For each $n \geq 0$, denote by $\mathscr{H}_{n}$ the subspace of $L^{2}\left(v_{\alpha}\right)$ generated by $\left\{\Psi_{A}, A \in \mathcal{E}_{n}\right\}$, so that $L^{2}\left(v_{\alpha}\right)=\bigoplus_{n \geq 0} \mathscr{H}_{n}$. Functions of $\mathscr{H}_{n}$ are said to have degree $n$.

For $n \geq 0$, denote by $\pi_{n}$ the projection of $L^{2}\left(v_{\alpha}\right)$ on $\mathscr{H}_{n}$. It follows from definition (2.3) of formal gradients that $\left\{\pi_{n} \Phi_{e}, e \in \mathbb{E}\right\}$ is a formal gradient if $\left\{\Phi_{e}, e \in \mathbb{E}\right\}$ is one:

LEMma 3.1. Let $\left\{\Phi_{e}, e \in \mathbb{E}\right\}$ be a formal gradient. Then, for every $n \geq 0$, $\left\{\pi_{n} \Phi_{e}, e \in \mathbb{E}\right\}$ is also a formal gradient.

PROOF. The proof is elementary. We verify that the projection $\pi_{n}$ commutes with the exchanges $\sigma^{e}$, which follows from a direct computation.

This lemma allows us to decompose a formal gradient into components of different degrees. We shall say that a formal gradient $\left\{\Phi_{e}, e \in \mathbb{E}\right\}$ has degree $n$ if each function $\Phi_{e}$ has degree $n$. The proofs of Theorems 2.1 and 2.3 are thus 
reduced to proving that a square-integrable formal gradient of degree $n$ can be approximated by gradients of local functions of degree $n$.

Let us compute $\sigma^{e} \Psi_{A}$. If $e=(x, y), \sigma^{e} \Psi_{A}=0$ unless $e$ connects $A$ and $A^{c}$. If $x \in A$ and $y \notin A$, then $\sigma^{e} \Psi_{A}=\Psi_{(A \backslash\{x\}) \cup\{y\}}$, and if $y \in A$ and $x \notin A$, then $\sigma^{e} \Psi_{A}=\Psi_{(A \backslash\{y\}) \cup\{x\}}$. In other words, $\sigma^{e} \Psi_{A}=\Psi_{\sigma^{e} A}$, with the obvious definition of $\sigma^{e} A=(A \backslash\{x\}) \cup\{y\}$ or $(A \backslash\{y\}) \cup\{x\}$. This suggests that we should turn the space $\mathbb{X}_{n}$ of subsets $A$ of cardinality $n$ in $\mathbb{X}$ into a graph with edges $\mathbb{E}_{n}$ by connecting two vertices $A$ and $B$ (i.e., subsets of $\mathbb{X}$ of cardinality $n$ ) in $\mathbb{X}_{n}$ with an edge if $B=(A \backslash\{x\}) \cup\{y\}$ for some $x \in A$ and $y \notin A$ that are connected by an edge in $\mathbb{X}$. The transition probabilities $p_{n}(A, B)$ on $\mathbb{X}_{n}$ are given by $p(x, y)$ if $B=(A \backslash\{x\}) \cup\{y\}$ for some $x \in A$ and $y \notin A$ and 0 otherwise.

Any local function $f$ of degree $n$ has a representation as

$$
f(\eta)=\sum_{A} f(A) \Psi_{A}(\eta)
$$

and

$$
\left(\sigma^{e} f\right)(\eta)=f\left(\sigma^{e} \eta\right)=\sum_{A} f(A) \sigma^{e} \Psi_{A}=\sum_{A} f\left(\sigma^{e} A\right) \Psi_{A} .
$$

The Dirichlet form $D(f)$ for a function of degree $n$ reduces to

$$
\frac{1}{2} \sum_{\substack{e \in \mathbb{E} \\ A \in \mathbb{X}_{n}}} p(e)\left|f\left(\sigma^{e} A\right)-f(A)\right|^{2} .
$$

The factor $1 / 2$ is explained by the sum over the unoriented edges. The total Dirichlet form of $f$ can be written as the sum

$$
\frac{1}{2} \sum_{n \geq 1} \sum_{\substack{e \in \mathbb{E} \\ A \in \mathbb{X}_{n}}} p(e)\left|\pi_{n} f\left(\sigma^{e} A\right)-\pi_{n} f(A)\right|^{2},
$$

where

$$
\pi_{n} f=\sum_{A \in \mathbb{X}_{n}} f(A) \Psi_{A}
$$

The approximation of any square-integrable formal gradient in $\mathbb{X}$ by gradients of local functions reduces to a similar property for each of the graphs $\mathbb{X}_{n}$. Note that for the purpose of the approximation result we can replace the Dirichlet form by any equivalent one.

Let $g$ be a connected graph with vertices $V=\{x\}$ and edges $\mathbb{E}=\{e\}$. We assume that the graph is countable and that there is an upper bound $D$ on the degree of all of its vertices. Let us pick for each edge an arbitrary orientation as the positive direction but keep the edges in both directions so we have the set $\widehat{\mathbb{E}}$ of oriented edges. Given a function $f: V \rightarrow \mathbb{R}$, we can define $g=\delta f: \widehat{\mathbb{E}} \rightarrow \mathbb{R}$ 
by $(\delta f)(e)=f(y)-f(x)$, where $(x, y)=e$ is an oriented edge. It is clear that $g: \widehat{\mathbb{E}} \rightarrow \mathbb{R}$ satisfies

$$
\sum_{e \in \ell} g(e)=0
$$

for any loop $\ell$ of oriented edges. We can turn the set of $g$ 's satisfying (3.2) into a Hilbert space $\mathscr{H}_{1}$ by defining

$$
\|g\|^{2}=\sum_{e \in \mathbb{E}}|g(e)|^{2}=\frac{1}{2} \sum_{e \in \widehat{\mathbb{E}}}|g(e)|^{2} .
$$

Given any $g$ satisfying (3.2), we can construct an $f$, unique up to an additive constant, such that $g=\delta f$. However, in general, we have no control on the growth of $f$ at $\infty$. We say that the graph has property $\mathbf{L D}$, if, for any given $g \in \mathscr{H}_{1}$, we can find a sequence of functions $f_{n}$, each of which vanishes outside a finite set of vertices, such that

$$
\lim _{n \rightarrow \infty}\left\|g-\delta f_{n}\right\|=0
$$

(cf. [3]). Not every graph has this property: a counterexample is given by the homogeneous tree of degree 3 (cf. [12], example (3.19), page 37).

The following lemma will allow us to deduce property LD for a graph from a closely related one.

LEMMA 3.2. Let $\mathrm{g}$ be a graph with the degree of its vertices bounded by $D$. Let $g_{1}$ be obtained from $g$ by deleting a set of vertices and retaining all the edges of $g$ that are contained in $g_{1}$. Assume that there are finite constants $c(k)$, depending only on $\left(k, g_{1}\right)$, such that any two vertices in $g_{1}$ that are connected by a path of $k$ edges in $g$ are connected by a path of at most $c(k)$ edges contained in $g_{1}$. Assume also that there is a bound $\ell$ such that any deleted vertex of $g$ is connected to an undeleted vertex in $g_{1}$ by a path of edges in $g$ of length at most $\ell$. Then if $g$ has property $\mathbf{L D}$ so does $g_{1}$.

PROOF. The proof proceeds along the following lines. Given a $g$ defined on the oriented edges of $g_{1}$ that satisfies (3.2), we can find $f$ on the vertices of $g_{1}$ such that $g(e)=(\delta f)(e)$. Assume that we can extend $f$ as a function $h$ defined on the vertices of $g$ in such a way that $\|\delta h\|_{g}^{2} \leq C\|g\|_{g_{1}}^{2}$. Because $g$ has property LD, we can find a sequence of functions $h_{n}$ defined on the vertices of $g$, each $h_{n}$ having finite support such that $\left\|\delta h_{n}-\delta h\right\|_{g}^{2} \rightarrow 0$ as $n \rightarrow \infty$. It follows that the restriction $f_{n}$ of $h_{n}$ to $g_{1}$ satisfies $\left\|\delta f_{n}-g\right\|_{g_{1}}^{2} \rightarrow 0$, proving that $g_{1}$ has the property $\mathbf{L D}$ as well.

This shows that all we need to do is to construct such an extension $h$ of $f$. In order to do this, let us proceed as follows. For any $x$ that is a deleted vertex, there is at least one vertex $x^{\prime}$ of $g_{1}$ within a distance $\ell$. Pick any one. Define $h(x)=f\left(x^{\prime}\right)$. 
If $e=(x, y)$ is a deleted edge, then $(\delta h)(e)=f\left(x^{\prime}\right)-f\left(y^{\prime}\right)$, where $x^{\prime}, y^{\prime}$, are vertices in $g_{1}$. The graph distance between $x^{\prime}$ and $y^{\prime}$ can be at most $2 \ell+1$ in $g$ and therefore at most $c(2 \ell+1)$ in $g_{1}$. It is clear that there is a constant $K=K(\ell, c(\cdot))$ such that all the edges involved in the path from $x^{\prime}$ to $y^{\prime}$ through the vertices of $g_{1}$ are within a distance $K$ from $x$ and $y$. For any edge $e$ let us define

$$
S_{K}(e)=\sum_{\substack{e^{\prime}: d\left(e, e^{\prime}\right) \leq K \\ e^{\prime} \in g_{1}}}\left|(\delta f)\left(e^{\prime}\right)\right|^{2},
$$

where the sum extends only over the edges of $g_{1}$ within a distance $K$ of $e$. We can define the distance between two edges as the largest of the four possible distances between the two pairs of vertices. They differ from one another by at most 2 . By an application of Schwarz's inequality and the crudest upper bound,

$$
|(\delta h)(e)|^{2} \leq c(2 \ell+1) S_{K}(e)
$$

and

$$
\|\delta h\|_{g}^{2} \leq c(2 \ell+1) \sum_{e} S_{K}(e)=C c(2 \ell+1)\|\delta f\|_{g_{1}}^{2},
$$

where

$$
C=\sup _{e}\left\{\left|e^{\prime}: d\left(e, e^{\prime}\right) \leq K\right|\right\} .
$$

Clearly, $C \leq C(K, D)=\left(D+D^{2}+\cdots+D^{K}\right)$, where $D$ is a bound on the degree of any vertex.

\section{LEMMA 3.3. Any symmetric random walk on $\mathbb{Z}^{d}$ has property $\mathbf{L D}$.}

PROOF. We can assume without loss of generality that the walk is the nearest neighbor one. On any graph, if $\delta f$ with $f$ having finite support is not dense in the Hilbert space $\mathscr{H}_{1}$, there will be a $g \in \mathscr{H}_{1}$ that is orthogonal to $\delta f$ for all finitely supported $f$. In other words, if we represent $g=\delta h$, after a summation by parts

$$
\sum_{e}(\delta h)(e)(\delta f)(e)=\sum_{e}\left(\delta^{*} \delta h\right)(e) f(e)=0 \quad \text { for all } f .
$$

For the operator

$$
L=\delta^{*} \delta,
$$

we have a solution of $L h \equiv 0$ with $\delta h \in \mathscr{H}_{1}$. We will show that this is impossible for the simple random walk on any $\mathbb{Z}^{d}$ (except for the trivial solution $h=0$ ). Define $h_{i}(x)=h\left(x+z_{i}\right)-h(x)$ where $z_{i}: 1 \leq i \leq d$ are the unit vectors in the coordinate directions. Then (3.2) implies $\left(e^{i \theta_{j}}-1\right) \hat{h}_{k}(\theta)=\left(e^{i \theta_{k}}-1\right) \hat{h}_{j}(\theta)$ a.e. for $1 \leq j<k \leq d$, where $\hat{h}_{j}$ is the Fourier transform of the $\ell_{2}$ function $h_{j}(x)$. 
Therefore there exists a function $u(\theta)$ on the $d$-torus such that, for $1 \leq j \leq d$, $\hat{h}_{j}(\theta)=\left(e^{i \theta_{j}}-1\right) u(\theta)$. It is easy to see that $L h=0$ now implies $k(\theta) u(\theta)=0$, where $k(\theta)=\sum_{j}\left(1-\cos \theta_{j}\right)>0$ for $\theta \neq 0$. Thus $\hat{h}_{j} \equiv 0$.

Clearly, the proof only depends on the property

$$
\sum_{x} p(x) e^{i \theta \cdot x}=\sum_{x} p(x) \cos (\theta \cdot x)<1
$$

for $\theta \neq 0$ which is the same as irreducibility.

LEMMA 3.4. Any symmetric exclusion process involving a finite number $k$ of particles on $\mathbb{Z}_{*}^{d}$ has property $\mathbf{L D}$.

PROOF. Without exclusion and allowing 0 , we have essentially a symmetric random walk on $\mathbb{Z}^{k d}$ which has property $\mathbf{L D}$. (We only have to deal with symmetric functions of the $k$ positions $x_{1}, \ldots, x_{k}$.) We now delete the set of vertices $A=$ $\left\{\left(x_{1}, \ldots, x_{k}\right)\right\}$, where either some $x_{i}=0$ or $x_{i}=x_{j}$ for some pair $i, j$. It is easy to verify that $A$ satisfies the conditions of Lemma 3.2 except when $d=1$ and the walk is the nearest neighbor one.

REMARK 3.5. Tom Liggett pointed out to us that this result can also be obtained by using coupling techniques due to Spitzer and Liggett, as presented in [9], Chapter 8, Section 1.

We have thus proved Theorems 2.1 and 2.3. We shall now prove Corollaries 2.2 and 2.4. Since the proofs are identical we will only deal with Corollary 2.2.

Proof OF Corollary 2.2. Consider a local function $h$ such that $\langle h\rangle_{\alpha} \equiv 0$ for any $\alpha \in[0,1]$. This is equivalent to $\langle h\rangle_{N, K} \equiv 0$ for any $K$ and $N$ sufficiently large. We define for such a function

$$
\begin{aligned}
\|h\|_{-1, \alpha}^{2} & =\sup _{f}\left\{2\langle h, f\rangle_{\alpha}-\langle f,(-\mathcal{L}) f\rangle_{\alpha}\right\}, \\
\|h\|_{-1, N, K}^{2} & =\sup _{f}\left\{2\langle h, f\rangle_{N, K}-\left\langle f,\left(-\mathcal{L}_{N}\right) f\right\rangle_{N, K}\right\} .
\end{aligned}
$$

In the first formula one uses only local functions $f$. The second formula is in a finite-dimensional space and we are free to use arbitrary functions $f$. We need to prove

$$
\lim _{\substack{N \rightarrow \infty \\ K_{N} /(2 N)^{d} \rightarrow \alpha}}\|h\|_{-1, N, K_{N}}^{2}=\|h\|_{-1, \alpha}^{2} .
$$


It is easy to see that

$$
\|h\|_{-1, \alpha}^{2} \leq \liminf _{\substack{N \rightarrow \infty \\ K_{N} /(2 N)^{d} \rightarrow \alpha}}\|h\|_{-1, N, K_{N}}^{2} .
$$

Indeed, by definition, for every $\varepsilon>0$, there exists a local function $f_{\varepsilon}$ such that

$$
\|h\|_{-1, \alpha}^{2} \leq 2\left\langle h, f_{\varepsilon}\right\rangle_{\alpha}-\left\langle f_{\varepsilon},(-\mathcal{L}) f_{\varepsilon}\right\rangle_{\alpha}+\varepsilon .
$$

Since $h$ and $f_{\varepsilon}$ are local functions, the right-hand side is equal to

$$
\begin{aligned}
& \lim _{\substack{N \rightarrow \infty \\
K_{N} /(2 N)^{d} \rightarrow \alpha}}\left\{2\left\langle h, f_{\varepsilon}\right\rangle_{N, K}-\left\langle f_{\varepsilon},(-\mathcal{L}) f_{\varepsilon}\right\rangle_{N, K}\right\}+\varepsilon \\
& =\lim _{\substack{N \rightarrow \infty \\
K_{N} /(2 N)^{d} \rightarrow \alpha}}\left\{2\left\langle h, f_{\varepsilon}\right\rangle_{N, K}-\left\langle f_{\varepsilon},\left(-\mathcal{L}_{N}\right) f_{\varepsilon}\right\rangle_{N, K}\right\}+\varepsilon \\
& \leq \liminf _{\substack{N \rightarrow \infty \\
K_{N} /(2 N)^{d} \rightarrow \alpha}}\|h\|_{-1, N, K}^{2}+\varepsilon .
\end{aligned}
$$

The reverse inequality is more demanding. Our goal is to prove that

$$
\limsup _{\substack{N \rightarrow \infty \\ K_{N} /(2 N)^{d} \rightarrow \alpha}}\|h\|_{-1, N, K_{N}} \leq\|h\|_{-1, \alpha} .
$$

It is more convenient to express the norms $\|h\|_{-1, N, K}$ and $\|h\|_{-1, \alpha}$ as

$$
\begin{aligned}
\|h\|_{-1, \alpha} & =\sup \left\{\langle h, f\rangle_{\alpha}:\langle f,-\mathcal{L} f\rangle_{\alpha} \leq 1\right\}, \\
\|h\|_{-1, N, K} & =\sup \left\{\langle h, f\rangle_{\alpha}:\left\langle f,-\mathcal{L}_{N} f\right\rangle_{N, K} \leq 1\right\},
\end{aligned}
$$

where the suprema are taken over $f$ from the unit balls in the corresponding Dirichlet spaces.

It is easy to see that the following lemma is equivalent to (3.6). In fact, we can choose $f_{N}$ as the solution of the variational problem (3.8) and applying the Lemma 3.6 we obtain (3.6).

Lemma 3.6. Let $K_{N} /(2 N)^{d} \rightarrow \alpha$. Assume that for some $A \geq 0$ we have a sequence of functions $f_{N}$ in $L_{2}\left(\mu_{N, K_{N}}\right)$ that satisfies

$$
\begin{array}{r}
\sup _{N}\left\langle f_{N},-\mathcal{L}_{N} f_{N}\right\rangle_{N, K_{N}} \leq 1, \\
\lim _{N \rightarrow \infty}\left\langle h, f_{N}\right\rangle_{N, K_{N}}=A .
\end{array}
$$

Then, for any $\varepsilon>0$, there is a local function $f_{\varepsilon}$ such that

$$
\left\langle f_{\varepsilon}, h\right\rangle_{\alpha} \geq A-\varepsilon \quad \text { and } \quad\left\langle f_{\varepsilon},-\mathcal{L} f_{\varepsilon}\right\rangle_{\alpha} \leq 1 .
$$


PROOF. Fix a positive integer $\ell$ large enough so that $\Lambda_{\ell}=\{-\ell, \ldots, \ell\}^{d}$ contains the support of $h$. Since $h$ has mean 0 with respect to all the canonical measures $\mu_{\ell, K}$, from linear algebra, $H=\mathscr{L}_{\Lambda_{\ell}}^{-1} h$ is a well-defined cylinder function. Here $\mathcal{L}_{\Lambda_{\ell}}$ is the generator of the exclusion process restricted to $\Lambda_{\ell}$. Notice the difference between $\mathscr{L}_{\Lambda_{\ell}}$ and $\mathscr{L}_{\ell}$. While the first generator stands for the exclusion process on the cube $\Lambda_{\ell}$, the second generator stands for the exclusion process on $\Lambda_{\ell}$ with periodic boundary conditions, that is, on $\mathbb{T}_{\ell}^{d}$. Since $h=\mathcal{L}_{\Lambda_{\ell}} H$, for any $N>\ell$,

$$
\left\langle h, f_{N}\right\rangle_{N, K_{N}}=-\frac{1}{2} \sum_{x, y \in \Lambda_{\ell}} p(y-x)\left\langle T_{x, y} f_{N}, T_{x, y} H\right\rangle_{N, K_{N}} .
$$

The summation in the above formula is carried over all sites in the cube $\Lambda_{\ell}$ (without periodic boundary condition). Fix a cube $\Lambda_{L}$ with $L \geq \ell$. Denote by $f_{N, L}$ the conditional expectation of $f_{N}$ given $\mathcal{F}_{\Lambda_{L}}=\sigma\left(\eta(x), x \in \Lambda_{L}\right)$. A simple computation shows that $E\left[T_{x, y} f_{N} \mid \mathcal{F}_{\Lambda_{L}}\right]=T_{x, y} E\left[f_{N} \mid \mathcal{F}_{\Lambda_{L}}\right]=T_{x, y} f_{N, L}$ for all bonds $\{x, y\}$ in $\Lambda_{\ell}$. Therefore, since $H$ is $\mathscr{F}_{\Lambda_{L}}$-measurable, taking conditional expectations with respect to $\mathcal{F}_{\Lambda_{L}}$, we obtain that the right-hand side of (3.11) is equal to

$$
-\frac{1}{2} \sum_{x, y \in \Lambda_{\ell}} p(y-x)\left\langle T_{x, y} f_{N, L}, T_{x, y} H\right\rangle_{N, K_{N}} .
$$

On the other hand, by Jensen's inequality and (3.9),

$$
\begin{aligned}
\frac{1}{2} \sum_{x, y \in \Lambda_{L}} p(x-y)\left\langle\left(T_{x, y} f_{N, L}\right)^{2}\right\rangle_{N, K_{N}} & \leq \frac{1}{2} \sum_{x, y \in \Lambda_{L}} p(x-y)\left\langle\left(T_{x, y} f_{N}\right)^{2}\right\rangle_{N, K_{N}} \\
& =\left\langle f_{N},-\mathcal{L}_{N} f_{N}\right\rangle_{N, K_{N}} \\
& \leq 1 .
\end{aligned}
$$

Since $f_{N, L}$ is a cylinder function, the state space $\{0,1\}^{\Lambda_{L}}$ is finite and we have a uniform bound on the $L^{2}\left(\mu_{N, K_{N}}\right)$ norms of the gradients of $f_{N, L}$, there exists a subsequence $N^{\prime}$ and a cylinder function $g_{L}$ such that the gradients of $f_{N^{\prime}, L}$ converge to the gradients of $g_{L}$ pointwise. On the other hand, by the equivalence of ensembles, as $N \uparrow \infty$ and $K_{N} /(2 N)^{d} \rightarrow \alpha$, the finite-dimensional marginals of the canonical measure $\mu_{N, K_{N}}$ converge to the corresponding marginals of $\mu_{\alpha}$. Therefore, as $N^{\prime} \uparrow \infty$ and as $K_{N} /\left(2 N^{\prime}\right)^{d} \rightarrow \alpha$, the expression in (3.12) converges to

$$
-\frac{1}{2} \sum_{x, y \in \Lambda_{\ell}} p(y-x)\left\langle T_{x, y} H, T_{x, y} g_{L}\right\rangle_{\alpha} .
$$

Moreover, since $T_{x, y} f_{N, L}$ converges to $T_{x, y} g_{L}$, by (3.13), 


$$
\begin{aligned}
\frac{1}{2} \sum_{x, y \in \Lambda_{L}} p(x-y)\left\langle\left\{T_{x, y} g_{L}\right\}^{2}\right\rangle_{\alpha} & =\frac{1}{4} \sum_{x, y \in \Lambda_{L}} p(x-y)\left\langle\left\{\nabla_{x, y} g_{L}\right\}^{2}\right\rangle_{\alpha} \\
& \leq 1
\end{aligned}
$$

uniformly in $L$.

For each $x, y$ in $\mathbb{Z}^{d}$ such that $y-x \in F$ where $F=\{z: p(z)>0\}, T_{x, y} g_{L}$ is a bounded sequence in $L^{2}\left(\mu_{\alpha}\right)$. There exists, therefore, a subsequence $L^{\prime}$ for which $T_{x, y} g_{L^{\prime}}$ converges weakly for each $x, y$ in $\mathbb{Z}^{d}$ such that $y-x \in F$. Denote by $\Phi_{x, y}$ the weak $L^{2}\left(\mu_{\alpha}\right)$ limit. By construction, as $L^{\prime} \uparrow \infty,(3.14)$ converges to

$$
-\frac{1}{2} \sum_{x, y \in \Lambda_{\ell}} p(x-y)\left\langle T_{x, y} H, \Phi_{x, y}\right\rangle_{\alpha} .
$$

Moreover, the collection of $L^{2}\left(\mu_{\alpha}\right)$ functions $\Phi_{x, y}$ satisfy conditions (2.3) of a formal gradient.

At this point we have proved that

$$
-\frac{1}{2} \sum_{x, y \in \Lambda_{\ell}} p(x-y)\left\langle T_{x, y} H, \Phi_{x, y}\right\rangle_{\alpha} \geq A
$$

and from (3.15) it follows that

$$
\frac{1}{4} \sum_{x, y \in \mathbb{Z}^{d}} p(x-y)\left\langle\Phi_{x, y}^{2}\right\rangle_{\alpha} \leq 1 .
$$

We can now apply Theorem 2.1 and approximate $\left\{\Phi_{x, y}\right\}$ by $\delta f_{\varepsilon}$, thereby establishing (3.10).

4. Approximations for the tagged particle. Given $1 \leq K \leq(2 N)^{d}$, denote by $\chi_{N, K}$ the space of configurations of $\chi_{N}$ with $K-1$ particles: $\mathcal{X}_{N, K}=$ $\left\{\xi \in \mathcal{X}_{N}, \sum_{x \in \mathbb{T}_{N, *}^{d}} \xi(x)=K-1\right\}$. Recall from Section 2 that $\mu_{N, K}$ stands for the uniform measure on $\mathcal{X}_{N, K}$ and that $\langle\cdot\rangle_{N, K}$ represents the expectation with respect to $\mu_{N, K}$.

Denote by $\mathcal{C}$ (resp. $\mathcal{C}_{0}$ ) the space of local functions (resp. local functions that have mean 0 with respect to all canonical measures). Thus a local function $h$ belongs to $\mathcal{C}_{0}$ provided $\langle h\rangle_{N, K}=0$ for all large enough $N$ and all $1 \leq K \leq(2 N)^{d}$. Examples of subspaces of $\mathcal{C}_{0}$ are $\mathcal{L}_{0} \mathcal{C}=\left\{\mathcal{L}_{0} h, h \in \mathcal{C}\right\}, \mathcal{L}_{\tau} \mathcal{C}=\left\{\mathcal{L}_{\tau} h, h \in \mathcal{C}\right\}$. Also $\left\{\psi_{\lambda}, \lambda \in \mathbb{R}^{d}\right\}$ defined by (2.8) are functions in $\mathcal{C}_{0}$.

On the space of local functions $\mathcal{C}$, consider the inner product $\langle\cdot, \cdot\rangle_{0, \alpha, 1}$ defined by

$$
\langle f, g\rangle_{0,1, \alpha}=\left\langle-\mathscr{L}_{0} f, g\right\rangle_{\alpha} .
$$

Here the index 0 signifies that only the jump part of the generator enters in the definition. Denote by $\mathscr{H}_{1, \alpha}^{0}$ the Hilbert space obtained by completing the space of local functions endowed with the above inner product and by $D_{0, \alpha}$ the Dirichlet 
form associated with the generator $\mathcal{L}_{0}$ and the probability measure $v_{\alpha}$, that is, $D_{0, \alpha}(f)=\left\langle-\mathcal{L}_{0} f, f\right\rangle_{\alpha}$. For a local function $f$, let

$$
\|f\|_{0,-1, \alpha}^{2}=\sup _{g \in \mathcal{C}}\left\{2\langle f, g\rangle_{\alpha}-D_{0, \alpha}(g)\right\}
$$

be the dual $H_{-1}$ norm.

REMARK 4.1. We could have defined the $H_{-1}$ norm as

$$
\|f\|_{0,-1, \alpha}=\sup \left\{\langle f, g\rangle_{\alpha}: D_{0, \alpha}(g) \leq 1\right\},
$$

where the supremum is taken over either local functions or functions in $L_{2}\left(v_{\alpha}\right)$ with Dirichlet norm bounded by 1 .

We denote by $\|f\|_{0,-1, \alpha}$ the $\mathscr{H}_{-1, \alpha}^{0}$ norm of $f$. It is known that every local function in $\mathcal{C}_{0}$ has finite $\mathscr{H}_{-1, \alpha}^{0}$ norm (cf. [5], Section 7.2). On the other hand, constants have infinite $\mathcal{H}_{-1, \alpha}^{0}$ norm. For two local functions $f, g$ with finite $\mathscr{H}_{-1, \alpha}^{0}$ norm, define by polarization the $\mathscr{H}_{-1, \alpha}^{0}$ inner product of $f, g$, denoted by $\langle f, g\rangle_{0,-1, \alpha}$. Let $\mathscr{H}_{-1, \alpha}^{0}$ be the Hilbert space induced by the local functions endowed with the inner product $\langle f, g\rangle_{0,-1, \alpha}$.

In a similar way, for each $N \geq 1$ and $1 \leq K \leq(2 N)^{d}$, we define the spaces $\mathscr{H}_{1, N, K}^{0}$ and $\mathscr{H}_{-1, N, K}^{0}$ and the norms $\|\cdot\|_{0,1, N, K}$ and $\|\cdot\|_{0,-1, N, K}$. According to Corollary 2.4, for any local function in $h \in \mathcal{C}_{0}$,

$$
\lim _{\substack{N \rightarrow \infty \\ K_{N} /(2 N)^{d} \rightarrow \alpha}}\|h\|_{0,-1, N, K_{N}}^{2}=\|h\|_{0,-1, \alpha}^{2} .
$$

Since the density $\alpha$ is fixed throughout this section, to keep the notation simple, for $n=-1$, 1 , we denote the norms $\|\cdot\|_{0, n, \alpha}$ and $\|\cdot\|_{n, \alpha}$ by $\|\cdot\|_{0, n}$ and $\|\cdot\|_{n}$ and we denote the inner product $\langle\cdot, \cdot\rangle_{\alpha}$ simply by $\langle\cdot, \cdot\rangle$. The main result of this section states that any $h \in \mathcal{C}_{0}$ can be approximated in $\|\cdot\|_{0,-1}$ norm by $\mathcal{L} f$ with a local function $f$. Notice that while the $H_{-1}$ norm corresponds to the generator $\mathcal{L}_{0}$, the generator that appears in front of $f$ is the full generator $\mathcal{L}=\mathscr{L}_{0}+\mathscr{L}_{\tau}$.

THEOREM 4.2. Let $h$ be a local function in $\mathfrak{C}_{0}$. For every $\varepsilon>0$, there exist local functions $u_{\varepsilon}, f_{\varepsilon}$ such that $h=\mathscr{L} f_{\varepsilon}+u_{\varepsilon}$ and $\left\|u_{\varepsilon}\right\|_{0,-1} \leq \varepsilon$.

Notice that $u_{\varepsilon}$ belongs to $\mathcal{C}_{0}$ because $\mathscr{L} f_{\varepsilon}$ belongs automatically to $\mathcal{C}_{0}$. Theorem 4.2 is based on the following two results. Fix a local function $h$ in $\mathcal{C}_{0}$. For each $\lambda>0$, let $u_{\lambda}$ be the solution of the resolvent equation

$$
\lambda u_{\lambda}-\mathcal{L} u_{\lambda}=-h .
$$

PROPOSITION 4.3. For every $\varepsilon>0$, there exists $\lambda_{0}>0$ such that $\left\|\mathcal{L} u_{\lambda}-h\right\|_{0,-1} \leq \varepsilon$ for all $\lambda<\lambda_{0}$. That is,

$$
\lim _{\lambda \rightarrow 0}\left\|\mathcal{L} u_{\lambda}-h\right\|_{0,-1}=0 .
$$


Proposition 4.4. Fix a local function $h$ in $\mathcal{C}_{0}$ and let $u_{\lambda}$ be the solution of the resolvent equation (4.2). For every $\varepsilon>0$ and every $\lambda>0$, there exists a local function $g_{\lambda, \varepsilon}$ such that $\left\|\mathscr{L}\left(u_{\lambda}-g_{\lambda, \varepsilon}\right)\right\|_{0,-1} \leq \varepsilon$.

Proof of Theorem 4.2. Since

$$
h=\mathscr{L} g_{\lambda, \varepsilon}+\mathscr{L}\left(u_{\lambda}-g_{\lambda, \varepsilon}\right)+\left(h-\mathscr{L} u_{\lambda}\right)
$$

and we can make

$$
\left\|\mathcal{L}\left(u_{\lambda}-g_{\lambda, \varepsilon}\right)\right\|_{0,-1}+\left\|\left(h-\mathcal{L} u_{\lambda}\right)\right\|_{0,-1}<\varepsilon
$$

by first picking a small $\lambda$ according to Proposition 4.3 to make the second term small and then by picking $\varepsilon$ small according to Proposition 4.4 so that the first term is small, Theorem 4.2 follows from Propositions 4.3 and 4.4 .

We now turn to the proofs of Propositions 4.3 and 4.4, which are based on the duality properties of the symmetric exclusion process explained in Section 3. Denote by $D_{0}(\cdot)$ the Dirichlet form associated with the jump part of the generator, by $D_{\tau}(\cdot)$ the Dirichlet form associated with the translation part of the generator and by $D(\cdot)$ the full Dirichlet form:

$$
\begin{aligned}
D_{0}(f) & =\left\langle f,\left(-\mathcal{L}_{0}\right) f\right\rangle, \\
D_{\tau}(f) & =\left\langle f,\left(-\mathcal{L}_{\tau}\right) f\right\rangle, \\
D(f) & =D_{0}(f)+D_{\tau}(f) .
\end{aligned}
$$

Recall the definition of the set $A_{x, y}$ given in Section 3 and notice that $\sigma^{x, y} \Psi_{A}=$ $\Psi_{A_{x, y}}$. In particular, if $f=\sum_{n \geq 1} \sum_{A \in \mathcal{E}_{n}} f(A) \Psi_{A}$,

$$
\begin{aligned}
D_{0}(f) & =\frac{1}{4} \sum_{x, y \in \mathbb{Z}_{*}^{d}} p(y-x) \int\left[f\left(\sigma^{x, y} \xi\right)-f(\xi)\right]^{2} d v_{\alpha} \\
& =\frac{1}{4} \sum_{n \geq 1} \sum_{x, y \in \mathbb{Z}_{*}^{d}} p(y-x) \sum_{A \in \mathcal{E}_{n}}\left\{f\left(A_{x, y}\right)-f(A)\right\}^{2} .
\end{aligned}
$$

Actually, if $F$ is any symmetric finite set that generates $\mathbb{Z}^{d}, D_{0}(f)$ is seen to be equivalent to the form

$$
\begin{aligned}
& \widehat{D}_{0}(f)=\frac{1}{2} \sum_{\substack{x, y \in \mathbb{Z}_{*}^{d} \\
y-x \in F}} \int\left[f\left(\sigma^{x, y} \xi\right)-f(\xi)\right]^{2} d v_{\alpha} \\
& =\frac{1}{2} \sum_{n \geq 1} \sum_{\substack{x, z \in \mathbb{Z}_{*}^{d} \\
y-x \in F}} \sum_{A \in \mathcal{E}_{n}}\left\{f\left(A_{x, y}\right)-f(A)\right\}^{2} \\
& =\sum_{n \geq 1} \sum_{e \in \mathbb{E}_{n}} \sum_{A \in \mathcal{E}_{n}}\left\{f\left(\sigma^{e} A\right)-f(A)\right\}^{2},
\end{aligned}
$$


where $\mathbb{E}_{n}$ is the set of edges in $\mathcal{E}_{n}$ with an edge $e$ connecting $A$ and $B$ present if $A=\sigma^{x, y} B$ for some $x, y \in \mathbb{Z}_{*}^{d}$ such that $y-x \in F$. A consequence of the equivalence of the two Dirichlet forms is their equivalence on all of the spaces $\mathscr{H}_{n}$ with constants that are independent of $n$.

On the other hand, for a set $A$ of $\varepsilon_{n}$, denote by $\tau_{z} A$ the set defined by

$$
\left(\tau_{z} A\right)= \begin{cases}A+z, & \text { if }-z \notin A, \\ (A+z) \backslash\{0\} \cup\{z\}, & \text { if }-z \in A,\end{cases}
$$

where $B+z$ is the set $\{x+z ; x \in B\}$. Therefore, in the case where $-z$ belongs to $A$, we first translate $A$ by $z$ (obtaining a new set that contains the origin) and then we remove the origin and we add the site $z$. Of course, $\tau_{z}: \mathcal{E}_{n} \rightarrow \mathcal{E}_{n}$ is a one-to-one function. We denote its inverse by $\tau_{z}^{-1}$, which is seen to be $\tau_{-z}$. A straightforward computation shows that $\tau_{z} \Psi_{A}=\Psi_{\tau_{z} A}$.

We now use the results in the Appendix to prove Proposition 4.3 along the lines of the proofs of Lemma 2.5 of [8] or Theorem 5.1 of [11].

Proof of Proposition 4.3. From the results in [6], one can show that

$$
\lim _{\lambda \rightarrow 0}\left\|\mathcal{L} u_{\lambda}-h\right\|_{-1}=0 .
$$

Our goal is to prove that

$$
\lim _{\lambda \rightarrow 0}\left\|\mathcal{L} u_{\lambda}-h\right\|_{0,-1}=0 .
$$

To this end, we write $u_{\lambda}=\sum_{n} u_{n, \lambda}$, where $u_{n, \lambda}=\pi_{n} u_{\lambda}$ is the projection of $u_{\lambda}$ into $\mathscr{H}_{n}$, the space functions of degree $n$ and a similar decomposition for $\mathcal{L} u_{\lambda}=v_{\lambda}=\sum_{n} v_{n, \lambda}$ as well as $h=\sum h_{n}$. Since $h$ is local, the decomposition for $h$ is only a finite sum, and since the decomposition is orthogonal in $\mathscr{H}_{-1, \alpha}^{0}$,

$$
\left\|\mathcal{L} u_{\lambda}-h\right\|_{0,-1}^{2}=\sum_{n \leq n_{0}}\left\|v_{n, \lambda}-h_{n}\right\|_{0,-1}^{2}+\sum_{n>n_{0}}\left\|v_{n, \lambda}\right\|_{0,-1}^{2}
$$

From (4.5) and Lemma A.1,

$$
\|f\|_{1}^{2} \leq C n\|f\|_{0,1}^{2},
$$

valid for $f \in \mathscr{H}_{n}$, it follows that, for each fixed $n$ and any function $u \in L^{2}$,

$$
\begin{aligned}
\left\|\pi_{n} u\right\|_{0,-1} & =\sup _{\substack{f \in \mathscr{H}_{n} \\
\|f\|_{0,1} \leq 1}}|\langle u, f\rangle| \leq \sup _{\|f\|_{1} \leq \sqrt{C n}}|\langle u, f\rangle| \\
& \leq \sqrt{C n}\|u\|_{-1} .
\end{aligned}
$$

Then we have

$$
\begin{aligned}
\left\|v_{n, \lambda}-h_{n}\right\|_{0,-1} & \leq \sqrt{C n}\left\|\mathcal{L} u_{\lambda}-h\right\|_{-1} \\
& \rightarrow 0 \quad \text { as } \lambda \rightarrow 0 .
\end{aligned}
$$


It is therefore sufficient to control

$$
\sum_{n} \sup _{\lambda}\left\|v_{n, \lambda}\right\|_{0,-1}<\infty .
$$

On the other hand, by the tridiagonal property of $\mathcal{L}$ and using Lemma A.1 and (4.6), we have

$$
\begin{aligned}
\left\|v_{n, \lambda}\right\|_{0,-1} & =\left\|\pi_{n} \mathcal{L}\left(u_{n-1, \lambda}+u_{n, \lambda}+u_{n+1, \lambda}\right)\right\|_{0,-1} \\
& \leq \sqrt{C n}\left\|\mathcal{L}\left(u_{n-1, \lambda}+u_{n, \lambda}+u_{n+1, \lambda}\right)\right\|_{-1} \\
& =\sqrt{C n}\left\|u_{n-1, \lambda}+u_{n, \lambda}+u_{n+1, \lambda}\right\|_{1} \\
& \leq C^{\prime} n\left\{\left\|u_{n-1, \lambda}\right\|_{0,1}+\left\|u_{n, \lambda}\right\|_{0,1}+\left\|u_{n+1, \lambda}\right\|_{0,1}\right\} .
\end{aligned}
$$

In view of estimate (A.5), the proof of the proposition is complete.

Proof OF PROPOSITION 4.4. Let $u_{\lambda}$ be the solution of the resolvent equation. In the proof of Proposition 4.3, we saw that

$$
\mathscr{L} u_{\lambda}=\mathscr{L}\left[\sum_{n \geq 1} u_{\lambda, n}\right]=\sum_{n \geq 1} \mathcal{L} u_{\lambda, n}
$$

can be evaluated term by term and the second sum converges rapidly in $\mathscr{H}_{0,-1}$. It is therefore enough to show that, if $g=\sum_{j=1}^{n} u_{\lambda, j}$, we can find for any given $\varepsilon$ a local function $g_{\varepsilon}$ such that $\left\|\mathcal{L}\left(g-g_{\varepsilon}\right)\right\|_{0,-1} \leq \varepsilon$. If $u \in \bigoplus_{j=1}^{n} \mathscr{H}_{j}$, with components $\left\{u_{j}\right\}$,

$$
\|\mathcal{L} u\|_{0,-1} \leq \sum_{j=1}^{n}\left\|\mathcal{L} u_{j}\right\|_{0,-1} \leq C \sum_{j=1}^{n} j\left\|u_{j}\right\|_{1} \leq C_{n}\|u\|_{0,1} .
$$

All we need to do now is to find a local function $g_{\varepsilon} \in \bigoplus_{j=1}^{n} \mathscr{H}_{j}$, such that

$$
\left\|g-g_{\varepsilon}\right\|_{0,1}<\varepsilon .
$$

Note that $g$ is in $L_{2}\left(v_{\alpha}\right)$ and the approximation can be done in each $\mathscr{H}_{j}$.

We now prove the main result of the article.

Proof of THEOREM 2.5. In view of (3.5), which holds with $\|\cdot\|_{-1, \alpha}$ in place of $\|\cdot\|_{0,-1, \alpha}$, to prove Theorem 2.5 , it is enough to show that

$$
\limsup _{\substack{N \rightarrow \infty \\ K_{N} /(2 N)^{d} \rightarrow \alpha}}\|h\|_{-1, N, K_{N}}^{2} \leq\|h\|_{-1, \alpha}^{2}
$$

for every local function $h$ in $\mathcal{C}_{0}$. Notice first that this statement is elementary if $h=\mathcal{L} h_{0}$ for some local function $h_{0}$. 
Fix $\varepsilon>0$. By Theorem 4.2, there exist local functions $f_{\varepsilon}$ and $u_{\varepsilon}$ such that $h=\mathscr{L} f_{\varepsilon}+u_{\varepsilon}$ and $\left\|u_{\varepsilon}\right\|_{0,-1, \alpha} \leq \varepsilon$. Let $N$ be larger than the supports of $\mathcal{L} f_{\varepsilon}$ and $u_{\varepsilon}$. In this case,

$$
\|h\|_{-1, N, K}=\left\|\mathcal{L} f_{\varepsilon}+u_{\varepsilon}\right\|_{-1, N, K} \leq\left\|\mathcal{L} f_{\mathcal{\varepsilon}}\right\|_{-1, N, K}+\left\|u_{\varepsilon}\right\|_{-1, N, K} .
$$

Since (4.7) is elementary for functions of type $\mathcal{L} g$, as $N \uparrow \infty$ and $K_{N} /(2 N)^{d} \rightarrow \alpha$, the first term on the right-hand side converges to $\left\|\mathcal{L} f_{\mathcal{E}}\right\|_{-1, \alpha}^{2}$. By Corollary 2.4, since $u_{\varepsilon}$ belongs to $\mathcal{C}_{0}$, as $N \uparrow \infty$ and $K_{N} /(2 N)^{d} \rightarrow \alpha$, the second term converges to $\left\|u_{\varepsilon}\right\|_{0,-1, \alpha}$, which is at most $\varepsilon$. Therefore,

$$
\limsup _{\substack{N \rightarrow \infty \\ K_{N} /(2 N)^{d} \rightarrow \alpha}}\|h\|_{-1, N, K_{N}} \leq\left\|\mathcal{L} f_{\varepsilon}\right\|_{-1, \alpha}+\varepsilon \leq\|h\|_{-1, \alpha}+2 \varepsilon .
$$

Since $\varepsilon>0$ is arbitrary we are done.

\section{APPENDIX}

We begin with a lemma.

LEMmA A.1. There exists a finite constant depending only on the transition probability $p$ such that

$$
D_{\tau}(f) \leq C n D_{0}(f)
$$

for all functions $f$ in $\mathscr{H}_{n}$.

Proof. We note that, on $\mathscr{H}_{n}, D_{0}(f)$ can be replaced by the expression

$$
\widehat{D}_{0}(f)=\frac{1}{2} \sum_{e \in \mathbb{E}_{n}} \sum_{A \in \mathcal{E}_{n}}\left\{f\left(\sigma^{e} A\right)-f(A)\right\}^{2}
$$

because of its equivalence to $D_{0}(f)$ which is, in fact, uniform in $n$. Here $\mathbb{E}_{n}$ is the set of edges in $\varepsilon_{n}$ connecting $A$ and $B$ if $A=\sigma^{x, y} B$ for some $x, y \in \mathbb{Z}_{*}^{d}$ such that $y-x \in F$. Recall that $F$ can be taken to be the set of points in $\mathbb{Z}^{d}$ where every coordinate is bounded by 2 in absolute value.

An easy calculation of

$$
\int(1-\xi(z))\left[f\left(\tau_{z} \xi\right)-f(\xi)\right]^{2} d \nu_{\alpha}
$$

for $f \in \mathscr{H}_{n}$ of the form $f=\sum_{|A|=n} f(A) \Psi_{A}$ leads to

$$
D_{\tau}(f)=\frac{1}{2} \sum_{z} \sum_{|A|=n} p(z) r_{z}(A)\left[f\left(\tau_{-z} A\right)-f(A)\right]^{2},
$$


with $r_{z}(A)=\alpha$ if $z \in A$ and $1-\alpha$ otherwise. Since $r_{z}(A) \leq 1$ and (A.1) is really a finite sum over $\{z: p(z)>0\}$, it is basically a question of estimating

$$
\sum_{A \in \mathcal{E}_{n}}\left(f\left(\tau_{-z} A\right)-f(A)\right)^{2}
$$

for each $z$. We can assume without loss of generality that $z=(1,0, \ldots)$, the unit vector in the direction of the first coordinate axis.

The problem is now reduced to the following: we are given a function $g: \mathcal{E}_{n} \rightarrow \mathbb{R}$. We think of $\mathcal{E}_{n}$ as a graph with edges $\mathbb{E}_{n}$. The Dirichlet form is

$$
\widehat{D}_{0}(g)=\frac{1}{2} \sum_{e \in \mathbb{E}_{n}}|(\delta g)(e)|^{2}
$$

and we are claiming an estimate of the form

$$
\frac{1}{2} \sum_{A \in \mathcal{E}_{n}}\left|g\left(\tau_{z} A\right)-g(A)\right|^{2} \leq C n \widehat{D}_{0}(g),
$$

with a constant independent of $n$. It is clear that for any $A$ and $B=\tau_{z} A$ one can move from $A$ to $B$ along the edges of the graph $\varepsilon_{n}$, using only the edges in $\mathbb{E}_{n}$. We will verify that we can assign a set of edges $\mathbb{E}_{A} \subset \mathbb{E}_{n}$ such that (i) for every $A$ one can use the edges of $\mathbb{E}_{A}$ to go from $A$ to $\tau_{z} A$, (ii) for any $A$ there are at most $n$ edges in $\mathbb{E}_{A}$ and (iii) the subsets $\left\{\mathbb{E}_{A}\right\}$ are mutually disjoint as $A$ varies over $\mathcal{E}_{n}$. Then it is easy to see that

$$
\left|g\left(\tau_{z} A\right)-g(A)\right|^{2}=\left|\sum_{e \in \mathbb{E}_{A}}(\delta g)(e)\right|^{2} \leq n \sum_{e \in \mathbb{E}_{A}}|(\delta g)(e)|^{2},
$$

and, summing over $A \in \mathcal{E}_{n}$, because the $\mathbb{E}_{A}$ are disjoint, we can establish the lemma.

To construct the paths from $A$ to $\tau_{z} A$, we totally order the points of $\mathbb{Z}_{*}^{d}$ by lexicographic ordering. We say that $z=\left(z_{1}, \ldots, z_{d}\right) \in \mathbb{Z}_{*}^{d}$ is positive if either $z_{1}>0$ or $z_{1}=0, z_{2}=0, \ldots, z_{j-1}=0$ and $z_{j}>0$ for some $j, 2 \leq j \leq d$. The total ordering declares $y>x$ if $y-x$ is positive. Let the set $A \in \mathcal{E}_{n}$ consist of the $n$ points $\left(x_{1}, x_{2}, \ldots, x_{n}\right)$ of $\mathbb{Z}_{*}^{d}$. We can assume that they are ordered so that $x_{1}>x_{2}>\cdots>x_{n}$. Then $\tau_{z} A=\left(x_{1}^{*}, \ldots, x_{n}^{*}\right)$, where $x_{j}^{*}=x_{j}+z$ unless $x_{j}=-z$ in which case $x_{j}^{*}=x_{j}+2 z=z$. We use the edges in $\mathbb{E}_{n}$ to shift successively each $x_{i}$ to $x_{i}^{*}$ starting from $x_{1}$ and proceeding in order and ending with shifting $x_{n}$. Any edge that is used goes from some $A_{1}$ to $A_{2}=\sigma^{x, x^{*}} A_{1}$. Since the shifts were made in lexicographic order we can determine without ambiguity which points of $A_{1}$ have already been shifted and which have not been. In other words, the paths from any two different $A$ to the corresponding $\tau_{y} A$ do not share a common edge. It is 
also clear that exactly $n$ edges are used. Any edge corresponds to $x^{*}-x=z$ or $2 z$, both of which are in $F$.

We want to look at the resolvent equation

$$
\lambda u_{\lambda}-\mathcal{L} u_{\lambda}=-h
$$

and obtain some decay estimates on the contributions to the Dirichlet form from terms of large degree. We follow essentially the method carried out in [8] or [11]. The lattice is $\mathbb{Z}_{*}^{d}$ and we have the Bernoulli measure $v_{\alpha}$ on $X=$ $\{0,1\}^{\mathbb{Z}_{*}^{d}}$. We consider the orthonormal basis $\left\{\Psi_{A}\right\}$ introduced in (3.1) and the decomposition of $L_{2}\left(v_{\alpha}\right)$ as $\bigoplus_{j} \mathscr{H}_{j}$. We will consider operators $\mathbb{T}$ acting on $L_{2}\left(v_{\alpha}\right)$ as scalar multiplication by $t(j)$ on $\mathcal{H}_{j}$. Assume that $t(j)$ is constant for $j \leq n_{1}$. These are the operators that commute with the projection operators $\pi_{j}$ onto the subspaces $\mathscr{H}_{j}$. The operator $\mathcal{L}$ can be written as $\mathcal{L}_{0}+\mathcal{L}_{\tau}$, the sum of the parts corresponding to the motions of untagged particles and the tagged particle. The operator $\mathscr{L}_{0}$ commutes with $\mathbb{T}$. Corresponding to the decomposition $L_{2}\left(v_{\alpha}\right)=\oplus \mathscr{H}_{j}$, we can write a block decomposition for $\mathcal{L}_{\tau}$ involving a tridiagonal block matrix $\mathfrak{L}_{i, j}$. If we write $u=\sum_{j} u_{j}$ where $u_{j}=\pi_{j} u$, then we can compute the commutator

$$
\begin{aligned}
{[\mathbb{T}, \mathcal{L}]=} & \mathbb{T} \mathcal{L}-\mathcal{L} \mathbb{T} \\
= & \sum_{j} t(j)\left[\mathfrak{L}_{j-1, j} u_{j-1}+\mathfrak{L}_{j, j} u_{j}+\mathfrak{L}_{j+1, j} u_{j+1}\right] \\
& -\sum_{j}\left[t(j-1) \mathfrak{L}_{j-1, j} u_{j-1}+t(j) \mathfrak{L}_{j, j} u_{j}+t(j+1) \mathfrak{L}_{j+1, j} u_{j+1}\right] \\
= & \sum_{j}\left[[t(j)-t(j-1)] \mathfrak{L}_{j-1, j} u_{j-1}+[t(j)-t(j+1)] \mathfrak{L}_{j+1, j} u_{j+1}\right] .
\end{aligned}
$$

We let $t(j) u_{j}=v_{j}$ and $t(j)-t(j-1)=s(j)$. From the reversibility of $\mathcal{L}_{\tau}$, we can conclude that

$$
\mathfrak{L}_{j-1, j}^{*}=\mathfrak{L}_{j, j-1} .
$$

We then use it to estimate the following quadratic form

$$
\begin{aligned}
& \left\langle[\mathbb{T}, \mathcal{L}] \mathbb{T}^{-1} \mathbb{T} u, \mathbb{T} u\right\rangle \\
& =\sum_{j}\left\langle[t(j)-t(j-1)] \mathfrak{L}_{j-1, j} u_{j-1}\right. \\
& \left.\quad+[t(j)-t(j+1)] \mathfrak{L}_{j+1, j} u_{j+1}, t(j) u_{j}\right\rangle \\
& =\sum_{j} \frac{s(j)}{t(j-1)}\left\langle\mathfrak{L}_{j-1, j} v_{j-1}, v_{j}\right\rangle
\end{aligned}
$$




$$
\begin{aligned}
& -\sum_{j} \frac{s(j+1)}{t(j+1)}\left\langle\mathfrak{L}_{j+1, j} v_{j+1}, v_{j}\right\rangle \\
= & \sum_{j} \frac{s(j)}{t(j-1)}\left\langle\mathfrak{L}_{j-1, j} v_{j-1}, v_{j}\right\rangle \\
& -\sum_{j} \frac{s(j+1)}{t(j+1)}\left\langle\mathfrak{L}_{j, j+1} v_{j}, v_{j+1}\right\rangle \\
= & \sum_{j} \frac{s(j)^{2}}{t(j-1) t(j)}\left\langle\mathfrak{L}_{j-1, j} v_{j-1}, v_{j}\right\rangle \\
= & \sum_{j} \frac{s(j)^{2}}{t(j-1) t(j)}\left\langle\mathcal{L}_{\tau} v_{j-1}, v_{j}\right\rangle \\
\leq & \sum_{j} \frac{s(j)^{2}}{t(j-1) t(j)} \sqrt{\left\langle-\mathcal{L}_{\tau} v_{j-1}, v_{j-1}\right\rangle} \sqrt{\left\langle-\mathcal{L}_{\tau} v_{j}, v_{j}\right\rangle} \\
\leq & \frac{1}{2} \sum_{j} \frac{s(j)^{2}}{t(j-1) t(j)}\left[\left\langle-\mathcal{L}_{\tau} v_{j-1}, v_{j-1}\right\rangle+\left\langle-\mathcal{L}_{\tau} v_{j}, v_{j}\right\rangle\right] .
\end{aligned}
$$

Since $-\mathscr{L}_{\tau}$ is symmetric and positive definite, the penultimate inequality is valid. We can now combine the last inequality of our calculation in (A.3) with Lemma A.1 to arrive at

$$
\left\langle[\mathbb{T}, \mathcal{L}] \mathbb{T}^{-1} \mathbb{T} u, \mathbb{T} u\right\rangle \leq \sum_{j} \frac{C}{2} \frac{j s(j)^{2}}{t(j-1) t(j)}\left[\left\langle-\mathcal{L}_{0} v_{j-1}, v_{j-1}\right\rangle+\left\langle-\mathcal{L}_{0} v_{j}, v_{j}\right\rangle\right] .
$$

Let us suppose that

$$
\sup _{j} \frac{C j s(j)^{2}}{t(j-1) t(j)} \leq \delta
$$

Then

(A.4) $\left\langle[\mathbb{T}, \mathcal{L}] \mathbb{T}^{-1} \mathbb{T} u, \mathbb{T} u\right\rangle \leq \delta\left\langle-\mathcal{L}_{0} v, v\right\rangle \leq \delta\langle-\mathcal{L} v, v\rangle=\delta\langle-\mathcal{L} \mathbb{T} u, \mathbb{T} u\rangle$

We start with the resolvent equation

$$
\lambda u_{\lambda}-\mathcal{L} u_{\lambda}=-h
$$

operate by $\mathbb{T}$ and take inner product with $v=\mathbb{T} u_{\lambda}$. We get

$$
\lambda\langle v, v\rangle-\left\langle\mathbb{T} \mathcal{L} \mathbb{T}^{-1} v, v\right\rangle=-\langle h, v\rangle,
$$


which can be rewritten using (A.4) as

$$
\begin{aligned}
\lambda\langle v, v\rangle-\langle\mathcal{L} v, v\rangle & =\left\langle[\mathbb{T} \mathcal{L}-\mathcal{L} \mathbb{T}] \mathbb{T}^{-1} v, v\right\rangle-\langle h, v\rangle \\
& \leq \delta\langle-\mathcal{L} v, v\rangle-\langle h, v\rangle .
\end{aligned}
$$

This implies the estimate

$$
\|v\|_{1}^{2}=\langle-\mathcal{L} v, v\rangle \leq(1-\delta)^{-1}|\langle h, v\rangle| \leq\|\mathbb{T} h\|_{-1}\|v\|_{1} \leq\|\mathbb{T} h\|_{0,-1}\|v\|_{1} .
$$

In particular,

$$
\sup _{\lambda>0}\left\|\mathbb{T} u_{\lambda}\right\|_{0,1}^{2} \leq(1-\delta)^{-1}\|\mathbb{T} h\|_{0,-1} .
$$

It is not hard to construct sequences $t(j)$ that satisfy

$$
\sup _{j} \frac{C j s(j)^{2}}{t(j-1) t(j)} \leq \delta
$$

and increase rapidly to $\infty$. We can get up to $e^{c \sqrt{n}}$ for some small $c>0$ that depends on $C$ and $\delta$. This a priori estimate can easily be formally established to show that for any local $h \in \mathscr{H}_{0,-1}$ there is a constant $C(k, h)$ such that

$$
D_{0}\left(\pi_{n} u_{\lambda}\right) \leq C(k, h) n^{-k}
$$

for all $\lambda>0$ and $n \geq 1$. Note that the mean zero property implies that $\pi_{0}\left(u_{\lambda}\right)=0$.

\section{REFERENCES}

[1] De Masi, A., Ferrari, P. A., Goldstein, S. and Wick, W. D. (1989). An invariance principle for reversible Markov processes: applications to random motions in random environments. J. Statist. Phys. 55 787-855.

[2] Giacomin, G., Olla, S. and Spohn, H. (2001). Equilibrium fluctuations for $\nabla \phi$ interface models. Ann. Probab. 29 1138-1172.

[3] GRIGOR' YAN, A. A. (1988). On Liouville theorems for harmonic functions with finite Dirichlet integral. Math. USSR-Sb. 60 485-504.

[4] Kesten, H. (1982). Percolation Theory for Mathematicians. Birkhäuser, Boston.

[5] Kipnis, C. and LANDiM, C. (1999). Scaling Limit of Interacting Particle Systems. Springer, Berlin.

[6] Kipnis, C. and VARADHAN, S. R. S. (1986). Central limit theorem for additive functionals of reversible Markov processes and applications to simple exclusion. Comm. Math. Phys. $1061-19$.

[7] LANDim, C. (1998). Decay to equilibrium in $L^{\infty}$ of asymmetric simple exclusion processes in infinite volume. Markov Process. Related Fields 4 517-534.

[8] Landim, C. and YaU, H. T. (1997). Fluctuation-dissipation equation of asymmetric simple exclusion processes. Probab. Theory Related Fields 108 321-356.

[9] Liggett, T. (1985). Interacting Particles Systems. Springer, Berlin.

[10] OsadA, H. and SAITOH, T. (1995). An invariance principle for non-symmetric Markov processes and reflecting diffusions in random domains. Probab. Theory Related Fields $10145-63$. 
[11] Sethuraman, S., Varadhan, S. R. S. and Yau, H. T. (2000). Diffusive limit of a tagged particle in asymmetric exclusion process. Comm. Pure Appl. Math. 53 972-1006.

[12] SoARdi, P. M. (1994). Potential Theory on Infinite Networks. Lecture Notes in Math. 1590. Springer, New York.

[13] VARADHAN, S. R. S. (1995). Self diffusion of a tagged particle in equilibrium for asymmetric mean zero random walks with simple exclusion. Ann. Inst. H. Poincaré Probab. Statist. 31 273-285

[14] VARAdHAN, S. R. S. (1994). Non-linear diffusion limit for a system with nearest neighbor interactions II. In Asymptotic Problems in Probability Theory: Stochastic Models and Diffusion on Fractals (K. D. Elworthy and N. Ikeda, eds.). 75-128. Wiley, New York.

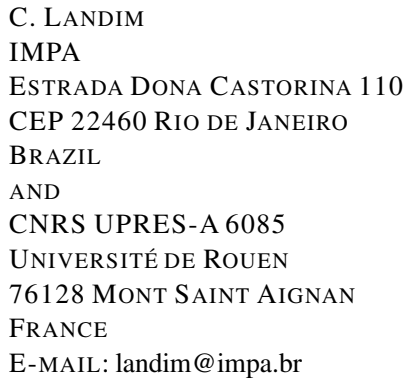

E-MAIL: landim@impa.br

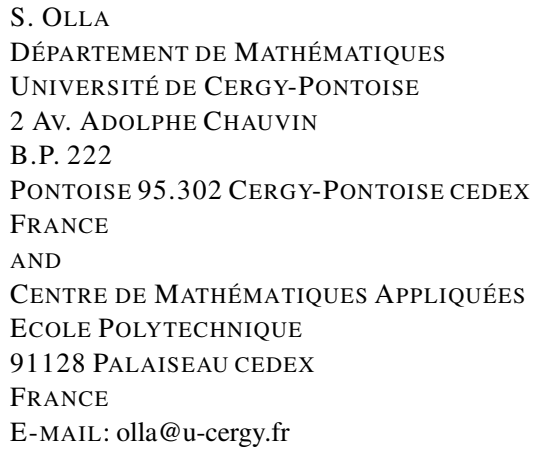

E-MAIL: olla@u-cergy.fr

S. R. S. VARADHAN

Courant Institute of Mathematical Sciences

NEW YORK UNIVERSITY

251 MERCER STREET

NEW York, NEW York 10012

E-MAIL: varadhan@cims.nyu.edu 